\title{
Impaired self-reported sleep quality improves with radiofrequency catheter ablation in patients with premature ventricular complexes
}

\author{
Mükremin Coskun', Mevlut Koc¹, Abdullah Orhan Demirtas', Muhammed Zubeyir Aslan², \\ Hilmi Erdem Sumbul², Hasan Koca', Tayfur Erdoğdu³, Yahya Kemal Icen \\ 1 Department of Cardiology, University of Health Sciences-Adana Health Practice and Research Center, Adana, Turkey \\ 2 Department of Internal Medicine, University of Health Sciences - Adana Health Practice and Research Center, Adana, Turkey \\ 3 Department of Cardiology, Osmaniye Public Hospital, Osmaniye, Turkey
}

\section{KEY WORDS}

poor sleep quality, premature ventricular contractions, radiofrequency catheter ablation
Correspondence to: Prof. Mevlut Koc, Department of Cardiology, University of Health Sciences-Adana Health Practice and Research Center, Dr. Mithat Özsan Bulvarı KışlaMah. 4522 Sok. No: 1 Yüreğir, Adana, Turkey, phone: +905062425989 , email: mevlutkoc78@yahoo.com Received: May 19, 2020. Revision accepted: July 9, 2020. Published online: July 17, 2020. Kardiol Pol. 2020; 78 (9): 899-905 doi:10.33963/KP.15519 Copyright by the Author(s), 2020

\section{ABSTRACT}

BACKGROUND The frequency of premature ventricular contractions (PVCs) increases in patients with poor sleep quality (PSQ).

AIMS The aim of this study was to evaluate PSQ in patients referred for radiofrequency catheter ablation (RFA) due to PVCs and to determine the effect of RFA on PSQ.

METHODS A total of 207 patients who were diagnosed with the PVC burden greater than $10 \%$ and underwent 3-dimensional RFA in our center were included in this cross-sectional study. Self-reported sleep quality was assessed in all patients in addition to a 24-hour Holter electrocardiogram before ablation and at 3-month follow-up. The effect of RFA on self-reported sleep quality in patients with PVCs was evaluated.

RESULTS Before RFA, 87\% of the study patients had PSQ. In those with PVCs, subjective sleep quality, sleep latency, sleep duration, sleep efficiency, sleep disturbances, daytime dysfunction, and the global Pittsburgh Sleep Quality Index (PSQI) improved after RFA $(P<0.001)$. Positive correlations were found between the PSQI and the total number of PVCs, PVC burden, PVC burden in the morning, PVC burden at midday, PVC burden in the evening, and PVC burden at nighttime $(P<0.01)$. In linear regression analysis, only the nighttime PVC burden was found to be related to the PSQI $(P=0.002, \beta=0.397)$.

CONCLUSIONS Poor sleep quality is common in patients with PVCs and it improves significantly after the RFA procedure. Poor sleep quality in patients with PVCs is closely related to the PVC burden at nighttime. Our study showed that, while evaluating PVCs, we also should suspect PSQ, especially due to PVCs.

INTRODUCTION Premature ventricular contractions (PVCs) are a very common arrhythmia in clinical practice. In the general population, it has been seen in $1 \%$ to $4 \%$ of patients on electrocardiography (ECG) and in $40 \%$ to $75 \%$ on 24-hour Holter ECG. ${ }^{1,2}$ This condition, which is generally benign, may cause sudden cardiac death in patients with structural heart disease. Numerous patients with PVCs are asymptomatic. Those symptomatic complain of palpitations, dizziness, near-syncope, dyspnea, chest pain, and fatigue. Apart from that, patients may experience syncope and present with signs of cardiomyopathy and heart failure as a result of nonsustained ventricular tachycardia (VT), sustained VT, or ventricular fibrillation, caused by PVCs. Triggering factors of PVCs include stress, dehydration, caffeine and alcohol consumption, medical treatment, hormonal cycles in women, and poor sleep quality (PSQ). ${ }^{3}$

In daily practice, PSQ is frequently associated with PVCs and it is often overlooked that PSQ may occur due to PVCs. In the routine evaluation of patients with PVCs, lifestyle changes 


\section{WHAT'S NEW?}

Treatment of poor sleep quality (PSQ) is recommended in the follow-up and treatment of patients with premature ventricular contractions (PVCs). Contrary to this general recommendation, it was shown for the first time in our study that the PVC burden status, especially the nighttime PVC burden, was the cause of PSQ. In addition, we showed that the treatment of PVCs with radiofrequency catheter ablation improved the PSQ status. Therefore, it was concluded that PVCs should be evaluated in terms of PSQ in addition to palpitations and other symptoms when assessing indications for ablation. Therefore, patients with frequent PVCs and accompanying PSQ can be referred to an arrhythmia center for radiofrequency catheter ablation.

\section{TABLE 1 Study inclusion and exclusion criteria}

\begin{tabular}{|c|c|}
\hline \multicolumn{2}{|r|}{ Inclusion criteria (in addition to symptomatic PVCs; at least 1 of the following criteria) } \\
\hline a & $\begin{array}{l}\text { Patients who had the PVC burden }>10 \% \text { on } 24 \text {-hour Holter } \\
\text { electrocardiography }\end{array}$ \\
\hline$b$ & $\begin{array}{l}\text { Patients who had clinically life-threatening rhythm disorder, syncope, or } \\
\text { abnormal } 24 \text {-hour Holter electrocardiography recordings (R-on-T } \\
\text { phenomenon and ventricular tachycardia) }\end{array}$ \\
\hline$c$ & Patients who had LVEF $<50 \%$ \\
\hline \multicolumn{2}{|c|}{ Exclusion criteria } \\
\hline a & Patients who had undergone a failed RFA procedure \\
\hline $\mathrm{b}$ & Recurrent PVCs at 3-month follow-up \\
\hline c & Hospitalization in the last month due to CAD or HF \\
\hline$d$ & $\begin{array}{l}\text { Patients who had insufficient PVCs for ablation mapping at the } \\
\text { procedure }\end{array}$ \\
\hline e & Known psychiatric disorders \\
\hline$f$ & Sleep apnea syndrome \\
\hline g & Chronic obstructive pulmonary disease \\
\hline $\mathrm{h}$ & Bone deformity involving the chest wall \\
\hline $\mathrm{i}$ & Active thyroid disease \\
\hline j & Malignancy \\
\hline k & Pregnancy or suspected pregnancy \\
\hline I & Chronic kidney disease \\
\hline $\mathrm{m}$ & Patients who received medical treatment that affected sleep quality \\
\hline $\mathrm{n}$ & Active infectious disease \\
\hline 0 & Patients who did not provide consent to participate in the study \\
\hline
\end{tabular}

Abbreviations: $C A D$, coronary artery disease; $H F$, heart failure; LVEF, left ventricular ejection fraction; PVC, premature ventricular contraction; RFA, radiofrequency catheter ablation

such as modifying sleep habits are recommended in the treatment of patients with PSQ. ${ }^{3}$ Our observations in daily practice showed that in patients treated with radiofrequency catheter ablation (RFA) due to symptomatic PVCs, sleep quality improved from the first day after the procedure and palpitation symptoms relieved. Palpitations, increased dyspnea, reduced functional capacity, anxiety, and frequent hospital admissions might affect sleep quality in patients with PVCs. We assumed that sleep quality could be impaired in patients with PVCs and that impaired sleep quality could increase the PVC burden and symptoms of PVCs. To the best of our knowledge, there has been no study evaluating the relationship between the PVC burden and PSQ so far.

Therefore, in our study, we aimed to evaluate PSQ in patients who underwent RFA due to PVCs and to determine the effect of RFA treatment on PSQ.

METHODS Study population In this cross-sectional study, patients with symptomatic PVCs who were resistant to pharmacological treatment and scheduled for an RFA procedure between August 2018 and April 2020 were screened at the arrhythmia center.

The study inclusion and exclusion criteria are shown in TABLE 1. In our study, a total of 282 patients were screened, but only 207 of them were included in the study. The regional hospital ethics committee approved the study (decision no., 2019/548). All of the included patients were informed about the study and provided informed consent to participate in it.

After inclusion, a detailed medical history was taken and physical examination was performed in all patients. Current medical treatments that patients received for PVCs were recorded. Serum glucose, creatinine, sodium, potassium, calcium, aspartate aminotransferase, alanine aminotransferase, uric acid, high-sensitivity C-reactive protein, $\mathrm{N}$-terminal fragment of the prohormone brain natriuretic peptide, total cholesterol, high-density lipoprotein cholesterol, low-density lipoprotein cholesterol, and triglyceride levels were measured using automated laboratory methods (Abbott Aeroset, Minneapolis, Minnesota, United States) and appropriate commercial kits (Abbott).

Two-dimensional echocardiography was performed with the EPIQ 7 device (Philips Healthcare, Andover, Massachusetts, United States). The M-mode examination in the parasternal long-axis view showed left ventricular (LV) diastolic and systolic dimensions. Left ventricular ejection fraction was calculated in the apical 4- and 2-chamber view by the modified Simpson method. ${ }^{4}$

Echocardiography and electrophysiology examinations in the study participants were evaluated by 2 experienced cardiologists. Both specialists had more than 5 years of experience in electrophysiological studies and performed at least 300 ablation procedures every year.

Evaluation of 24-hour Holter electrocardiograms Twenty-four-hour Holter ECG recordings (SEER TM 1000 GE Medical Systems, Milwaukee, Wisconsin, United States) before RFA of all patients included in the study 
TABLE 2 Demographic, clinical, laboratory, medical treatment, and echocardiographic characteristics of 207 study patients with premature ventricular complexes

\begin{tabular}{|c|c|}
\hline Characteristic & Value \\
\hline Age, y, median (IQR) & $51(37-62)$ \\
\hline Male / female sex, $\mathrm{n}$ & $113 / 94$ \\
\hline Hypertension, n (\%) & $52(25)$ \\
\hline Diabetes, n (\%) & $47(23)$ \\
\hline Current smoking status, $\mathrm{n}(\%)$ & $48(23)$ \\
\hline CAD, n (\%) & $38(18)$ \\
\hline Systolic BP, mm Hg, mean (SD) & $123(12)$ \\
\hline Diastolic BP, mm Hg, mean (SD) & $80(10)$ \\
\hline BMI, kg/m², mean (SD) & $28.1(3.28)$ \\
\hline Blood urea nitrogen, mg/dl, mean (SD) & $29.9(8.9)$ \\
\hline Creatinine, mg/dl, mean (SD) & $0.75(0.18)$ \\
\hline Sodium, mg/dl, mean (SD) & $138(3.5)$ \\
\hline Potassium, mg/dl, mean (SD) & $4.24(0.48)$ \\
\hline Aspartate aminotransferase, $\mathrm{u} / \mathrm{l}$, median (IQR) & $21(17-28)$ \\
\hline Alanine aminotransferase, u/l, median (IQR) & $21(16-28)$ \\
\hline Total cholesterol, mg/dl, median (IQR) & $190(164-212)$ \\
\hline Low-density lipoprotein cholesterol, mg/dl, median (IQR) & $128(102-144)$ \\
\hline High-density lipoprotein cholesterol, mg/dl, median (IQR) & $42(36-49)$ \\
\hline Triglycerides, mg/dl, median (IQR) & $150(109-209)$ \\
\hline Uric acid, mg/dl, mean (SD) & $4.82(0.95)$ \\
\hline NT-proBNP, pg/ml, median (IQR) & $70(37-148)$ \\
\hline$\beta$-Blocker, n (\%) & $126(61)$ \\
\hline Calcium channel blocker, n (\%) & $20(10)$ \\
\hline Amiodarone, $\mathrm{n}(\%)$ & $35(17)$ \\
\hline Propafenone, n (\%) & $10(5)$ \\
\hline LVDD, mm, mean (SD) & $47.5(5.1)$ \\
\hline LVSD, mm, mean (SD) & $31.4(5.3)$ \\
\hline LVEF, \%, mean (SD) & $58.6(7.9)$ \\
\hline LVEF < $50 \%, n(\%)$ & $14(7)$ \\
\hline Right ventricular PVCs, n (\%) & $122(59)$ \\
\hline Left ventricular PVCs, n (\%) & $85(41)$ \\
\hline
\end{tabular}

SI conversion factors: to convert blood urea nitrogen to $\mathrm{mmol} / \mathrm{l}$, multiply by 0.357 ; creatinine to $\mu \mathrm{mol} / \mathrm{l}$, by 88.4 ; sodium to $\mathrm{mmol} / \mathrm{l}$, by 1 ; potassium to $\mathrm{mmol} / \mathrm{l}$, by 1 ; aspartate aminotransferase and alanine aminotransferase to $\mu \mathrm{kat} / \mathrm{I}$, by 0.0167 ; total cholesterol, low-density lipoprotein cholesterol, high-density lipoprotein cholesterol to $\mathrm{mmol} / \mathrm{l}$, by 0.0259 ; triglycerides to $\mathrm{mmol} / \mathrm{l}$, by 0.0113; uric acid to $\mathrm{mmol} / \mathrm{l}$, by 0.0595 .

Abbreviations: BMI, body mass index; BP, blood pressure; LVDD, left ventricular diastolic dimension LVSD, left ventricular systolic dimension; NT-proBNP, N-terminal fragment of the prohormone brain natriuretic peptide; others, see TABLE 1

were examined. All recordings perceived as inaccurate by the device were corrected. Maximum heart rate (HR), minimum HR, mean HR, total 24-hour HR, and the total number of PVCs during 24 hours were recorded. The PVC burden was defined as the percentage of PVCs in the total number of QRS complexes during a 24-hour period.

\section{Self-reported sleep quality assessment} The Pittsburgh Sleep Quality Index (PSQI) consisting of 7 components and 19 specific questions evaluating subjective sleep quality, sleep latency (how long it takes to fall asleep), sleep duration, sleep efficacy (how long a person is asleep in comparison to their time spent in bed), sleep disorders (temperature, cold, noise, pain, and nocturia), sleep medications, and daytime dysfunction (sleepiness and concentration disorders) were used to evaluate sleep quality. ${ }^{5}$ Each component was scored between 0 and 3 points, with a high score indicating low sleep quality. These scores were summed to obtain the global measure of subjective sleep quality (0-21 points). Low PSQI values indicated better subjective sleep quality. The recommended clinical PSQI cutoff value for good subjective sleep quality was 5 . $\mathrm{Pa}$ tients with a PSQI greater than 5 were considered as having PSQ.

Electrophysiological study, mapping, induction, and radiofrequency ablation protocol The right ventricular outflow tract and the right ventricle were mapped with a standard femoral approach, and the LV outflow tract and the LV were mapped with a retrograde transaortic approach. In some of the LV papillary muscle-induced PVCs, the transition to the left atrium was achieved by septostomy and PVC mapping was performed using an antegrade approach. The procedure was continued in patients with an adequate PVCs frequency. Afterwards, PVCs were mapped with a 3-dimensional mapping system (CARTO 3, Biosense Webster, Diamond Bar, Irwindale, California, United States). The earliest local electrical signals were marked. These points were ablated with an irrigated RFA catheter delivering between 30 to 50 watts of power. After PVCs had disappeared, safety was provided by energizing ablation for approximately 3 minutes until the impedance value was lower than 110 ohms.

Clinical follow-up and determination of recurrence The patients who underwent RFA were initially followed up in the first month and then in 3-month intervals. The presence of PVCs on ECG or 24-hour Holter ECG recordings with sustained or nonsustained VT or PVCs of more than 1000 beats/day were regarded as recurrence. In patients with recurrence, RFA was recommended to those with a PVC burden greater than $10 \%$, and patients with that smaller than $10 \%$ were encouraged to continue medical treatment. Self-reported sleep quality (SRSQ) assessment was repeated in patients without PVC recurrence at 3-month follow-up. 
TABLE 3 Data on 24-hour Holter electrocardiography monitoring in 207 study patients with premature ventricular complexes

\begin{tabular}{ll} 
Parameter & Value \\
\hline Maximum heart rate, bpm & $132(21)$ \\
\hline Minimum heart rate, bpm & $47(9.2)$ \\
\hline Mean heart rate, bpm & $74(12)$ \\
\hline Total beat, $\mathrm{n}$ & $101058(91930-101058)$ \\
\hline Total PVCs, $\mathrm{n}$ & $16873(12030-16873)$ \\
\hline PVC burden, \% & $17(13-24)$ \\
\hline PVC burden in the morning, \% & $16(11-24)$ \\
\hline PVC burden at midday, \% & $16(12-26)$ \\
\hline PVC burden in the evening, \% & $18(12-24)$ \\
\hline PVC burden at nighttime, \% & $16(11-21)$ \\
\hline
\end{tabular}

Data are presented as mean (SD) or median (interquartile range). The premature ventricular contraction burden was defined as the percentage of premature ventricular contractions in the total number of QRS complexes during a 24-hour period.

Abbreviations: see TABLE 1

TABLE 4 Localization of premature ventricular contractions in patients who underwent successful radiofrequency catheter ablation

\section{PVC localization}

Patients, n (\%)

PVCs originating from the RV

Total

$122(59)$

\begin{tabular}{ll}
\hline RVOT anterior wall & $42(20)$ \\
\hline RVOT anterolateral wall & $10(4.8)$ \\
\hline RVOT posterior wall & $20(9.7)$ \\
\hline RVOT posteroseptal wall & $34(16)$ \\
\hline Pulmonary artery region & $5(2.4)$ \\
\hline Para-Hisian region & $3(1.4)$ \\
\hline Tricuspid annulus region & $5(2.4)$ \\
\hline Moderator band region & $3(1.4)$ \\
\hline PVCs originating from the LV & \\
\hline Total & $85(41)$ \\
\hline LVOT left coronary cusp & $22(11)$ \\
\hline LVOT aortomitral junction & $14(7)$ \\
\hline LVOT right coronary cusp & $17(8)$ \\
\hline LVOT right-left coronary cusp junction & $15(7)$ \\
\hline LV summit region & $5(2.4)$ \\
\hline Mitral annulus region & $7(3.4)$ \\
\hline Papillary muscle region & $5(2.4)$ \\
\hline
\end{tabular}

Data are presented as number (percentage).

Abbreviations: LV, left ventricle; LVOT, left ventricular outflow tract; RV, right ventricle; RVOT, right ventricular outflow tract; others, see TABLE 1
Statistical analysis All statistical analyses were performed using the SPSS 23.0 software package for Windows, version 23.0 (IBM Corp., Armonk, New York, United States). Whether the distribution of continuous variables was normal or not was evaluated with the Kolmogorov-Smirnov test. Continuous variables were expressed as mean (SD) or median (interquartile range). Categorical variables were expressed as numbers and percentages. The Cohen $\mathrm{x}$ and intraclass correlation coefficient were used for categorical and continuous data, respectively, to evaluate the inter- and intraobserver variability of SRSQ and 24-hour Holter ECG parameters. To compare the change in parameters of SRSQ before and after RFA observed at baseline and 6 months, a paired sample $t$ test and the McNemar test were applied. A $P$ value less than 0.05 was considered significant. Parameters associated with the PSQI were determined with the univariate Pearson correlation analyses. In multiple linear regression analysis, parameters showing the closest association with the PSQI were identified.

RESULTS Patients who underwent RFA due to PVCs and who did not have recurrent PVCs diagnosed based on 24-hour Holter ECG in the controls were included in the study. The SRSQ test was performed in all patients included in the study. The Cohen $\mathrm{k}$ and intraclass correlation coefficient values for interobserver variability were over $90 \%$ for all 24-hour Holter ECG recordings and SRSQ parameters $(P<0.001$ for all comparisons).

Demographic, clinical, laboratory, echocardiography, and 24-hour Holter electrocardiography data Demographic, clinical, laboratory, medical treatment, and echocardiographic characteristics of the study population are shown in tABLE2. The results of 24-hour Holter ECG recordings of the study patients are presented in TABLE3. Although PVC frequency in the patients included in the study was higher than $10 \%$, the mean PVC frequency was found to be $17 \%$ (13\%-24\%) (TABLE3). The localization of PVCs in patients who underwent RFA with the 3-dimensional mapping method is shown in TABLE 4. In 16 patients, PVCs from the regions of both the right ventricular outflow tract and the left ventricular outflow tract were eliminated with RFA, and the earliest intracardiac monitoring record in these patients was regarded as the localization of PVCs. Recurrence was detected in 18 patients $(7.1 \%)$ who underwent RFA. Eleven of them $(4.4 \%)$ were reablated and the others were followed up and received medication.

Self-reported sleep quality before and after radiofrequency ablation The change in parameters of SRSQ before and after the RFA 
TABLE 5 Change in parameters of self-reported sleep quality before and after radiofrequency catheter ablation in 207 patients with premature ventricular complexes

\begin{tabular}{llll} 
Parameter & Before RFA & After RFA & P value \\
Subjective sleep quality & $2.26(0.62)$ & $0.94(0.45)$ & $<0.001$ \\
\hline Sleep latency & $1.87(0.52)$ & $1.07(0.38)$ & $<0.001$ \\
\hline Sleep duration & $1.40(0.51)$ & $0.99(0.26)$ & $<0.001$ \\
\hline Sleep efficiency & $1.31(0.48)$ & $1.01(0.48)$ & $<0.001$ \\
\hline Sleep disturbances & $1.28(0.47)$ & $0.72(0.46)$ & $<0.001$ \\
\hline Daytime dysfunction & $2.14(0.69)$ & $\begin{array}{l}\text { Median (IQR), } \\
1(0.7-1.3)\end{array}$ & $<0.001$ \\
\hline Global PSQI & $10.3(2.33)$ & $5.34(1.69)$ & $<0.001$ \\
\hline PSQI $>5, \mathrm{n}(\%)$ & $180(87)$ & $25(12)$ & $<0.001$ \\
\hline
\end{tabular}

Data are presented as mean (SD) unless otherwise indicated.

Abbreviations: IQR, interquartile range; PSQI, Pittsburgh Sleep Quality Index; others, see TABLE 1

TABLE 6 Parameters associated with the Pittsburgh Sleep Quality Index before radiofrequency catheter ablation

\begin{tabular}{|c|c|c|c|c|}
\hline \multirow[t]{2}{*}{ Parameter } & \multicolumn{2}{|c|}{ Univariate analysis } & \multicolumn{2}{|c|}{ Multiple linear regression analysis } \\
\hline & $P$ value & $r$ & $P$ value & $\beta$ \\
\hline Total PVCs & $<0.001$ & 0.337 & 0.64 & 0.061 \\
\hline PVC burden & $<0.001$ & 0.421 & 0.29 & 0.095 \\
\hline $\begin{array}{l}\text { PVC burden in } \\
\text { the morning }\end{array}$ & $<0.001$ & 0.399 & 0.14 & 0.105 \\
\hline PVC burden at midday & $<0.001$ & 0.386 & 0.10 & 0.112 \\
\hline $\begin{array}{l}\text { PVC burden in } \\
\text { the evening }\end{array}$ & $<0.001$ & 0.350 & 0.50 & 0.090 \\
\hline PVC burden at nighttime & $<0.001$ & 0.418 & 0.002 & 0.397 \\
\hline
\end{tabular}

$R_{A d j u s t e d}^{2}=0.449$ and $P<0.001$ in multivariate analyses. The premature ventricular contraction burden is a ratio of the number of premature ventricular contractions to the total number of QRS complexes.

Abbreviations: see TABLE1

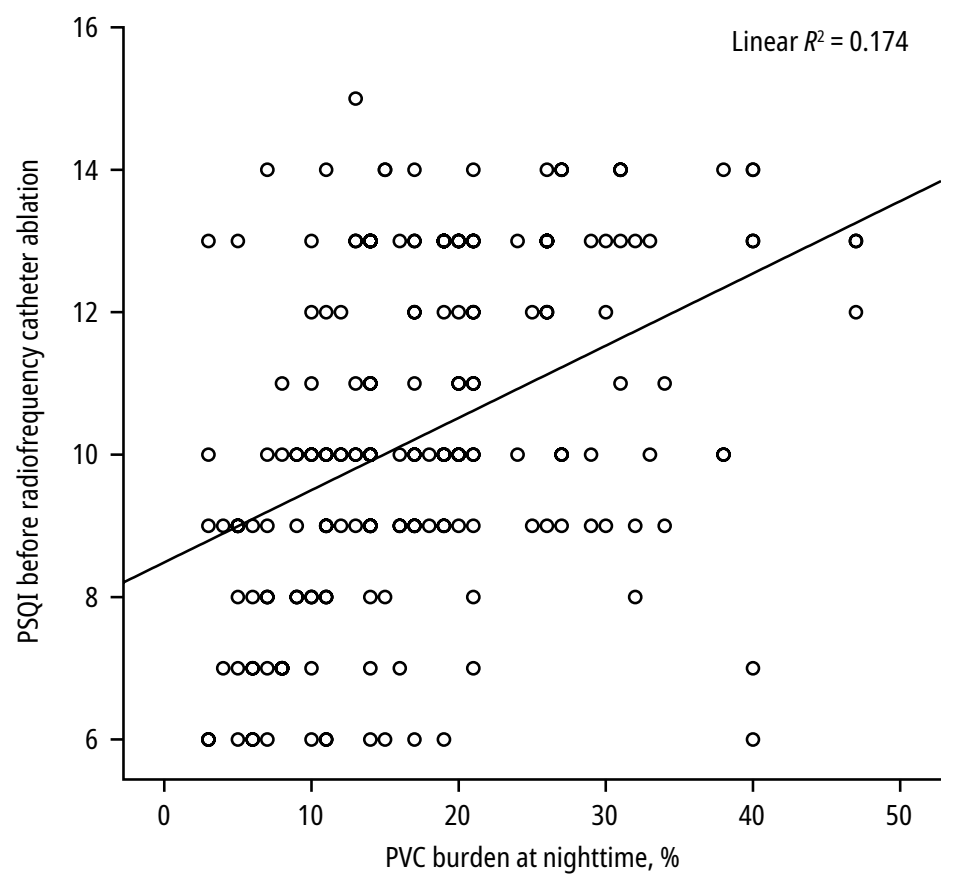

FIGURE 1 Scatter plot of the relationship between the Pittsburgh Sleep Quality Index and premature ventricular complexes at nighttime procedure in the study patients are shown in TABLE5. In those with PVCs, subjective sleep quality, sleep latency, sleep duration, sleep efficiency, sleep disturbances, daytime dysfunction, and the global PSQI were significantly decreased after RFA (TABLE 5). A total of $79.2 \%$ of the patients with symptomatic PVCs had PSQ before RFA and their PSQ rates decreased significantly (by $12 \%$ ) at 3 months after RFA (TABLE 5).

Determination of Pittsburgh Sleep Quality Index-related parameters Correlation analysis was performed between the PSQI and 24-hour Holter ECG recordings (TABLE 6). Linear regression analysis was applied to the parameters associated with the PSQI in the univariate analysis. As a result of this analysis, only the PVC burden at nighttime was found to be related to the PSQI (TABlE6). The closest relationship between the mean PSQI and the PVC burden at nighttime is shown in FIGURE1.

DISCUSSION Our study revealed several relevant findings: 1) sleep quality is impaired in patients with symptomatic PVCs; 2) in these patients, PSQ is closely related to PVCs at night; 3) treatment of patients with symptomatic PVCs with RFA significantly improves impaired sleep quality (60\%). To the best of our knowledge, our study is the first one to evaluate sleep quality in patients with symptomatic PVCs and the PVC burden greater than $10 \%$, indicating that it is associated with the PVC burden at nighttime.

Patients with PVCs are primarily evaluated based on medical history and physical examination. Then, advanced techniques such as ECG, 24-hour Holter ECG monitoring, echocardiography, cardiac magnetic resonance imaging, exercise test, fluorodeoxyglucose cardiac positron emission tomography, and electrophysiological studies are used for the diagnosis and treatment of PVCs. ${ }^{3}$ Twelve-lead ECG is used to determine the localization of PVCs. The PVC burden and life-threatening R-on-T phenomenon, VT, or ventricular fibrillation are assessed with 24-hour Holter ECG. Particularly, LV function and additional cardiac valve and wall abnormalities are detected by echocardiography. Also, echocardiography affects the choice of an approach during RFA treatment. The exercise test verifies the suspicion of ischemic heart disease and measures the frequency of PVCs or fatal dysrhythmia inducibility with exercise. These examinations can also enable healthcare professionals to explain whether PVCs are idiopathic or associated with structural heart diseases. Sleep quality in patients with PVCs is not routinely evaluated. However, it is known that sleep duration and quality are very meaningful factors in cardiovascular diseases. According to previous studies, short sleep time (shorter than 5 to 
6 hours) or long sleep time (longer than 9 hours) adversely affects cardiovascular events. ${ }^{3,6,7}$ Atrial fibrillation (AF) ablation results in significant improvement of symptoms and quality of life. ${ }^{8-9}$ Sleep quality has been found to be impaired in patients with arrhythmic events or AF, and AF treatment has been shown to improve sleep quality. ${ }^{7,10-13}$ According to a study by Szymanski et al, ${ }^{13}$ approximately half of the patients with AF have PSQ. The most important causes of PSQ in this patient population include continuous sympathoexcitation due to increased sympathetic activity and irregular ventricular response.

The RFA procedure in patients with PVCs has been shown to improve quality of life and is a cost-effective treatment method. ${ }^{14}$ However, the effect of successful treatment of PVCs on sleep quality in this patient cohort remains unclear. In our study, the most relevant causes of PSQ in patients with PVCs were as follows: 1) increased symptomatic activity in patients with PVCs; 2) a ventricular pause after PVCs, followed by a stronger normal beat, and change in nervous system regulation aimed at compensating for this condition; 3) some PVCs becoming more prominent, especially with decreasing HR at night; 4) nighttime environmental stimuli are less frequent, so patients sense their own heartbeat; and 5) increased anxiety caused by patients' learning that they have PVCs. In our study, all these causes could not be explored or the reason for PSQ cannot be explained clearly. However, the close relationship between PSQ and the nighttime PVC burden has shown us that there is a clear association between increasing PVCs, especially at night, and sleep disorders. In our study, despite the successful treatment of PVCs, the ongoing sleep disorder was similar to sleep disorders present in approximately $20 \%$ of the general population, regardless of any discomfort. ${ }^{15}$

Limitations Our study had some relevant limitations. The most important one was that there has been no other study which our recent data can be compared with. There is only a single study that shows a relationship between $\mathrm{AF}$ and PSQ. ${ }^{10}$ Another limitation of our study was the association between the frequency or presence of PVCs and PSQ regarded as "possible" or "hypothetical." We performed the sleep quality assessment using a subjective algorithm. More reliable results could be obtained if sleep quality was evaluated by following up patients for a few days in the sleep laboratory before and after the procedure. We did not know patients' sleep status, especially at the time before they had PVCs or palpitations. More reliable findings could be obtained if we could evaluate the sleep habits of the study patients in a long period of time, rather than in a certain limited time interval. Another important limitation was that patients who had a known psychiatric disorder or depression or who used medication for these reasons were excluded from the study, but not every patient underwent psychiatric evaluation. Of note, sleep disorders are common especially in psychiatric diseases. Another important reason for the improvement of sleep quality in patients after PVC ablation may be the absence of PVCs and a positive mood. In our study, the successful assessment was made at 3 months after PVC ablation. Different results could be obtained if the evaluation was performed at 1 month or 6 months.

Conclusions Self-reported sleep quality is poor in patients with the PVC burden greater than $10 \%$, and sleep quality improves significantly after a successful RFA procedure. Our study showed that it should be kept in mind that poor sleep is one of the symptoms observed in patients with PVCs. At the first evaluation of patients with PCVs, sleep quality should be assessed in addition to other known complaints, especially palpitations. Particularly if the patient begins to complain of palpitations, they should be asked whether they have noted deterioration in sleep quality at night and the association between palpitations and sleep disturbances should be examined. Since the data on PVCs and sleep quality obtained in our study are the first findings on this issue in the literature, they need to be supported by further research, in which other sleep evaluations should be performed, involving various patient groups.

\section{ARTICLE INFORMATION}

\section{CONFLICT OF INTEREST None declared.}

OPEN ACCESS This is an Open Access article distributed under the terms of the Creative Commons Attribution-NonCommercial-NoDerivatives 4.0 International License (CC BY-NC-ND 4.0), allowing third parties to download articles and share them with others, provided the original work is properly cited, not changed in any way, distributed under the same license, and used for noncommercial purposes only. For commercial use, please contact the journal office at kardiologiapolska@ptkardio.pl.

HOW TO CITE Coskun M, Koc M, Demirtas A0, et al. Impaired self-reported sleep quality improves with radiofrequency catheter ablation in patients with premature ventricular complexes. Kardiol Pol. 2020; 78: 899-905. doi:10.33963/KP.15519

\section{REFERENCES}

$1 \mathrm{Ng} \mathrm{GA}$. Treating patients with ventricular ectopic beats. Heart. 2006; 92: 1707-1712.

2 Kennedy HL, Whitlock JA, Sprague MK, et al. Long-term follow-up of asymptomatic healthy subjects with frequent and complex ventricular ectopy. $\mathrm{N}$ Engl J Med. 1985; 312: 193-197.

3 Gorenek B, Fisher JD, Kudaiberdieva G, et al. Premature ventricular complexes: diagnostic and therapeutic considerations in clinical practice: a state-of-the-art review by the American College of Cardiology Electrophysiology Council. J Interv Card Electrophysiol. 2020; 57: 5-26.

4 Sahn DJ, DeMaria A, Kisslo J, Weyman A. Recommendations regarding quantitation in M-mode echocardiography: results of a survey of echocardiographic measurements. Circulation. 1978; 58: 1072-1083.

5 Buysse DJ, Reynolds CF, Monk TH, et al. The Pittsburgh Sleep Quality Index: a new instrument for psychiatric practice and research. Psychiatry Res. 1989; 28: 193-213.

6 Wojdyła-Hordyńska A, Kowalski 0, Hordyński GJ, et al. The effect of radiofrequency catheter ablation of frequent premature ventricular complexes and arrhythmia burden on left ventricular function. Kardiol Pol. 2017; 75: 698-704.

7 Morovatdar N, Ebrahimi N, Rezaee R, et al. Sleep duration and risk of atrial fibrillation: a systematic review. J Atr Fibrillation. 2019; 11: 2132. 
8 Hindricks G, Sepehri Shamloo A, Lenarczyk R, et al. Catheter ablation of atrial fibrillation: current status, techniques, outcomes and challenges. Kardiol Pol. 2018; 76: 1680-1686.

9 Lomper K, Sławuta A, Dudek K, et al. Psychometric evaluation of the Polish version of the arrhythmia-specific questionnaire in tachycardia and arrhythmia: a new tool for symptom and health related quality of life assessment. Kardiol Pol. 2019; 77: 541-552.

10 Kayrak M, Gul EE, Aribas A, et al. Self-reported sleep quality of patients with atrial fibrillation and the effects of cardioversion on sleep quality. Pacing Clin Electrophysiol. 2013; 36: 823-829.

11 Brignole M, Gianfranchi L, Menozzi C, et al. Assessment of atrioventricular junction ablation and DDDR mode-switching pacemaker versus pharmacological treatment in patients with severely symptomatic paroxysmal atrial fibrillation: a randomized controlled study. Circulation. 1997; 96: 2617-2624.

12 Wokhlu A, Monahan KH, Hodge DO, et al. Long-term quality of life after ablation of atrial fibrillation the impact of recurrence, symptom relief, and placebo effect. J Am Coll Cardiol. 2010; 55: 2308-2316.

13 Szymanski FM, Filipiak KJ, Karpinski G, et al. Occurrence of poor sleep quality in atrial fibrillation patients according to the EHRA score. Acta Cardiol. 2014; 69: 291-296.

14 Huang CX, Liang J], Yang B, et al. Quality of life and cost for patients with premature ventricular contractions by radiofrequency catheter ablation. Pacing Clin Electrophysiol. 2006; 29: 343-350.

15 Ohayon MM, Carskadon MA, Guilleminault C, Vitiello MV. Meta-analysis of quantitative sleep parameters from childhood to old age in healthy individuals: developing normative sleep values across the human lifespan. Sleep. 2004; 27: 1255-1273. 